\title{
Management strategies for infants with coarctation and an associated ventricular septal defect
}

\author{
J. William Gaynor, MD
}

From the Cardiac Center at The Children's
Hospital of Philadelphia, Philadelphia, $\mathrm{Pa}$.

Received for publication April 19, 2001; accepted for publication April 24, 2001.

Address for reprints: J. William Gaynor, MD, Pediatric Cardiothoracic Surgery, The Children's Hospital of Philadelphia, 34th St \& Civic Center Blvd, Philadelphia, PA 19104.

J Thorac Cardiovasc Surg 2003;125:S87-9

Originally published in $\mathrm{J}$ Thorac Cardiovasc Surg 2001;122:424-6.

Copyright (C) 2003 by The American Association for Thoracic Surgery

0022-5223/2003\$30.00+0

doi: $10.1067 / \mathrm{mtc} .2003 .238$

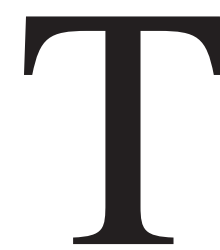

he optimal management strategy for patients with aortic coarctation and an associated ventricular septal defect (VSD) remains controversial. The patients are heterogeneous, with considerable variation in the morphology of the aortic arch, as well as variation in the characteristics of the VSD. Additional levels of left ventricular outflow tract obstruction are often present. Systemic perfusion may be dependent on ductal patency, and surgical repair is usually necessary in the neonatal period or early infancy. A variety of therapeutic strategies have been proposed, including coarctation repair alone with later VSD closure, if necessary; coarctation repair with pulmonary artery banding followed by delayed VSD closure and debanding; and single-stage repair of both defects. Multiple techniques have also been used for arch reconstruction in these patients, including subclavian flap aortoplasty, patch aortoplasty, and resection with end-to-end anastomosis. Not surprisingly, there is an ongoing debate over the best technique for arch reconstruction and the timing of VSD closure. Most reports contain relatively small numbers of patients and focus on the short-term outcome of a specific strategy, usually in terms of operative mortality and early recoarctation. Little data exist concerning the long-term outcome of these patients, specifically persistent hypertension, ventricular function, adverse neurodevelopmental sequelae, and the incidence of reintervention other than for recoarctation.

In 1994, the Congenital Heart Surgeons Society reported results of a multiinstitutional study evaluating 326 symptomatic neonates with coarctation either with or without an associated VSD. ${ }^{1}$ A VSD was present in 155 of these infants, and 153 underwent surgical repair. This report provided an important snapshot of management strategies and outcomes at the participating institutions but did not resolve questions concerning the optimal management strategy. For neonates having a moderate or large VSD, repair of the coarctation plus pulmonary artery banding was associated with both the highest non-risk-adjusted and risk-adjusted survival. Single-stage repair of both defects was associated with the lowest non-risk-adjusted survival. However, the authors noted that there was uncertainty as to the optimal strategy because the $P$ values for the differences in survival were generally greater than .05. On the basis of this report, as well as concerns over use of deep hypothermic circulatory arrest (DHCA) during the arch repair and the risks of recoarctation, many institutions have continued to advocate a multistage repair strategy. Nevertheless, as the authors of the Congenital Heart Surgeons Society study noted, there are potential important advantages to single-stage repair.

Factors that must be considered when evaluating management strategies for these infants include the following: (1) whether the VSD is sufficiently large to result in congestive heart failure after coarctation repair alone; (2) whether the VSD is of a type that is likely to close spontaneously; (3) whether proximal arch hypoplasia is present; and (4) whether the subaortic area and aortic valve are of adequate size. Advocates of coarctation repair alone note that many VSDs close spontaneously, and thus a second operation can be avoided. However, many infants with moderate or large VSDs will have persistent congestive heart failure after isolated coarctation repair and may remain ventilator dependent. Closure of the VSD is often necessary during the same hospitalization. In addition, certain types of VSD (malalignment type with posterior deviation of the infundibular septum and conal septal hypoplasia 
types) never undergo spontaneous closure, and thus patients with these defects would be committed to a second operation. Coarctation repair in conjunction with pulmonary artery banding has been advocated to prevent congestive heart failure caused by the VSD. However, such a strategy requires that all patients undergo a second operation for VSD closure and pulmonary artery debanding or debanding alone if the VSD closes spontaneously. Single-stage repair of both defects through a median sternotomy has the advantages of correcting all hemodynamic abnormalities at the initial procedure with no obligate need for reoperation. In addition, proximal arch hypoplasia and subaortic stenosis are not uncommon in these infants, and repair through a median sternotomy allows augmentation of the entire aortic arch and subaortic muscle resection, if necessary.

In the current issue of the Journal, Isomatsu and associates $^{2}$ report early and intermediate-term outcomes after 2-stage repair of coarctation and an associated VSD in 79 patients. All patients underwent initial coarctation repair with subclavian flap aortoplasty. VSD closure and removal of the pulmonary artery band were performed at a mean interval of 10 months after coarctation repair. The short- and intermediate-term outcomes were excellent, with a low operative mortality (2/79) and 4 late deaths. Two of the late deaths, however, occurred during the second operation for VSD closure, and the other 2 late deaths were caused by congestive heart failure after the second-stage operation. The estimated freedom from recoarctation was $90 \%$ at 10 years. These outstanding results confirm that 2-stage repair of coarctation with an associated VSD can be performed with excellent early and intermediate-term outcomes. The authors state that they prefer a 2-stage repair strategy because of potential mortality associated with single-stage repair, avoidance of DHCA, decreased incidence of recoarctation, and a decreased need for blood transfusion.

Other investigators continue to advocate single-stage repair. Several recent reports have demonstrated that excellent early results can be obtained with a single-stage repair strategy. Ishino and colleagues ${ }^{3}$ reported outcome after single-stage repair with use of isolated cerebral perfusion to avoid DHCA during the arch repair. They compared singlestage repair in 11 infants (5 with a hypoplastic arch) with 2 -stage repair in 13 patients (4 with a hypoplastic arch). There were no operative deaths and 1 late death in each group. They noted no neurologic complications; however, formal neurodevelopmental assessments were not performed. At a mean follow-up of 20 months for the singlestage repair, no patients had recurrent arch obstruction. The mean follow-up for the patients undergoing multistage repair is longer (67 months), and 1 patient had recoarctation. Haas and colleagues ${ }^{4}$ reported outcomes of 21 premature ( $<36$ weeks' gestational age) or low-birth-weight $(<3000$ g) infants undergoing single-stage repair of interrupted aor- tic arch (10) or coarctation with an associated VSD (11). There were 3 (14\%) early deaths. Subaortic obstruction was present in 2 of the patients with coarctation. At a mean follow-up of 33 months, recurrent arch obstruction had developed in 2 patients.

At the Children's Hospital of Philadelphia, we have adopted a policy of single-stage repair for infants with coarctation and a moderate or large VSD and recently reported the outcome of 25 infants after single-stage repair. ${ }^{5}$ Arch reconstruction was performed by means of either patch aortoplasty (21) or end-to-end anastomosis (4). There was $1(4 \%)$ operative death and 1 late death from sepsis in a patient with complete absence of the thymus. Freedom from reintervention for recurrent coarctation was $75 \% 2$ years after the operation. The incidence of reintervention was the same for patch reconstruction of the arch (20\%) as for resection with end-to-end anastomosis (25\%). Several patients, however, have had progressive subaortic stenosis, and 4 have subsequently undergone the Ross-Konno procedure.

These reports demonstrate that both multistage and single-stage repair of coarctation with an associated VSD can be accomplished with excellent early outcomes and acceptable rates of recoarctation, even in very small infants. There are limitations to these studies, however, and many questions remain unanswered. The number of patients in each study was relatively small. None of the studies directly compare single-stage versus multistage repair in a prospective randomized fashion. Early mortality in the recent series is low for both single-stage and multistage repair. Although recoarctation is an important complication, the incidence of recoarctation is acceptable for both techniques. In addition, management of recurrent arch obstruction has been greatly simplified by increasing use of balloon dilation. Thus, mortality and recoarctation may not be the most appropriate outcome measures to differentiate between the 2 strategies in the current era. Finally, there is a lack of long-term follow-up data.

The long-term results for neonatal corrective surgery for complex congenital heart defects are not known. Neonatal repair of congenital heart defects has been advocated on the basis of the hypothesis that early correction of cyanosis and abnormal hemodynamics will result in a superior outcome over the lifetime of the child. For example, there is increasing evidence that early primary repair of tetralogy of Fallot results in an improved outcome compared with initial palliation and late complete repair. ${ }^{6}$ Single-stage repair of coarctation and an associated VSD is similarly appealing because all hemodynamic lesions are corrected without an obligate need for reoperation. However, because the early results of single-stage and multistage repair are similar in terms of mortality and early recoarctation, long-term studies with assessment of the incidence of persistent hypertension, 
ventricular dysfunction, and adverse neurodevelopmental sequelae are necessary to determine whether one strategy is clearly superior. Concerns over possible neurologic injury during arch reconstruction are appropriate; however, all children who undergo cardiac surgery are at risk for adverse neurodevelopmental sequelae irrespective of whether DHCA is used. ${ }^{7}$ The use of isolated cerebral perfusion to avoid DHCA during arch reconstruction is appealing. However, it is important to realize that despite the increasing enthusiasm for the technique of isolated cerebral perfusion and its intellectual appeal, no studies of neurodevelopmental outcome have been performed to confirm that the technique is indeed superior to DHCA. In addition to long-term outcomes, the role of new techniques in the management of these infants must be evaluated. A recent report described the use of absorbable material for pulmonary artery banding in infants with a VSD that is likely to close spontaneously, thus avoiding the need for a second operation. ${ }^{8}$ In some infants with coarctation associated with a VSD and left ventricle outflow tract obstruction at the valvular and subvalvular levels, the neonatal Ross-Konno procedure may be indicated. The effect of new and evolving techniques cannot be predicted.

Coarctation with an associated VSD is a relatively uncommon lesion, and single institutions will be unable to accumulate a sufficient number of patients to address these issues in a reasonable time period. On the basis of the current evidence in the literature, it cannot be definitively stated that either single-stage or multistage repair for patients with coarctation and an associated VSD results in a superior long-term outcome. Each strategy has advantages and disadvantages; however, the early results for both are good. In addition, there is continuing controversy over the best technique for arch reconstruction. A long-term, multiinstitutional, prospective, randomized study with a large number of patients will be needed to determine whether one strategy is associated with a better long-term outcome.

\section{References}

1. Quaegebeur JM, Jonas RA, Weinberg AD, Blackstone EH, Kirklin JW. Outcomes in seriously ill neonates with coarctation of the aorta: a multiinstitutional study. J Thorac Cardiovasc Surg. 1994;108:84154.

2. Isomatsu Y, Imai Y, Shin'oka T, Aoki M, Sato K. Coarctation of the aorta and ventricular septal defect: Should we perform a single-stage repair? J Thorac Cardiovasc Surg. 2001;122:524-8.

3. Ishino K, Kawada M, Irie H, Kino K, Sano S. Single-stage repair of aortic coarctation with ventricular septal defect using isolated cerebral and myocardial perfusion. Eur J Cardiothorac Surg. 2000;17:538-42.

4. Haas F, Goldberg CS, Ohye RG, Mosca RS, Bove EL. Primary repair of aortic arch obstruction with ventricular septal defect in preterm and low birth weight infants. Eur J Cardiothorac Surg. 2000;17:643-7.

5. Gaynor JW, Wernovsky G, Rychik, J, Rome JJ, DeCampli WM, Spray TL. Outcome following single-stage repair of coarctation with ventricular septal defect. Eur J Cardiothorac Surg. 2000;20:1-6.

6. Van Arsdell GS, Maharaj GS, Tom J, Rao VK, Coles JG, Freedom RM, et al. What is the optimal age for repair of tetralogy of Fallot? Circulation. 2000;102(Suppl):III-123-9.

7. Bellinger DC, Wypij D, Kuban KCK, Rappaport LA, Hickey PR, Wernovsky G, et al. Developmental and neurological status of children at 4 years of age after heart surgery with hypothermic circulatory arrest or low-flow cardiopulmonary bypass. Circulation. 1999;100: 526-32.

8. Bonnet D, Patkaï J, Tamisier D, Kachaner J, Vouhé P, Sidi D. A new strategy for the surgical treatment of aortic coarctation associated with ventricular septal defect in infants using an absorbable pulmonary artery band. J Am Coll Cardiol. 1999;34:866-70. 